\title{
High-isolation Double-pole-double-throw Switch with Eighth-wavelength Transmission Lines for Wireless Sensors
}

\author{
Si-Da Tang ${ }^{*}$ and Kai-Li Wang ${ }^{2}$ \\ ${ }^{1}$ School of Internet of Things Engineering, Jiangnan University, \\ No. 1800, Lihu Avenue, Wuxi, Jiangsu 214122, China \\ ${ }^{2}$ Toshiba Corporation, 1-1, Shibaura 1-Chome, Minato-ku, Tokyo 105-8001, Japan \\ (Received February 9, 2020; accepted August 28, 2020)
}

Keywords: DPDT, eighth wavelength, high isolation, switch

In this paper, we present the implementation of a novel structure of a double-pole-double-throw (DPDT) switch with eighth-wavelength transmission lines. The switch consists of eight diodes, eight eighth-wavelength transmission lines, and four frequency-optimized matching lines. It can be used as a single-pole-double-throw (SPDT) switch when one of the ports is terminated with a characteristic impedance of $50 \Omega$ to the ground. By using eighth-wavelength transmission lines, the isolation of the switch is highly improved. The performance characteristics of the traditional quarter-wavelength SPDT and DPDT have also been evaluated for comparison with the proposed design. Measured results show that the insertion loss reaches a value as high as $1.65 \mathrm{~dB}$, and the isolation is $30 \mathrm{~dB}$ at a frequency of $2.4 \mathrm{GHz}$. The size of the switch is nearly the same as that of the conventional quarter-wavelength DPDT switch. This novel switch can be used in wireless sensor applications.

\section{Introduction}

Software-defined radio (SDR) is a radio communication system that covers not only existing wireless standards, but also future standards that might be a more attractive prospect. Generally, most of the physical layer functions are software-defined, ${ }^{(1)}$ and wireless sensors are one of the most important applications. ${ }^{(2)}$ Basic SDR requires an RF front-end system for signal transmission. As shown in Fig. 1, a double-pole-double-throw (DPDT) switch is usually used in RF front-end systems to enable multi-antenna channel switching between the transmitter and the receiver. Figure 2(a) shows a conventional back-to-back structure DPDT switch consisting of two single-pole-double-throw (SPDT) switches. The problem of the structure is its high insertion loss. Another DPDT structure is of the ring type shown in Fig. 2(b), which is composed of four single-pole-single-throw (SPST) switches. The insertion loss is good because the signal passes through only one switch from an antenna to either a transmitter or a receiver.

As the connection between the elements of a wireless system, the switch requires high isolation. Many switches using the traveling-wave concept with quarter-wavelength transmission lines have been reported. ${ }^{(3-5)}$ In Ref. 3, the switch combined off-state shunt transistors and

*Corresponding author: e-mail: tomtsd@163.com https://doi.org/10.18494/SAM.2021.2826 


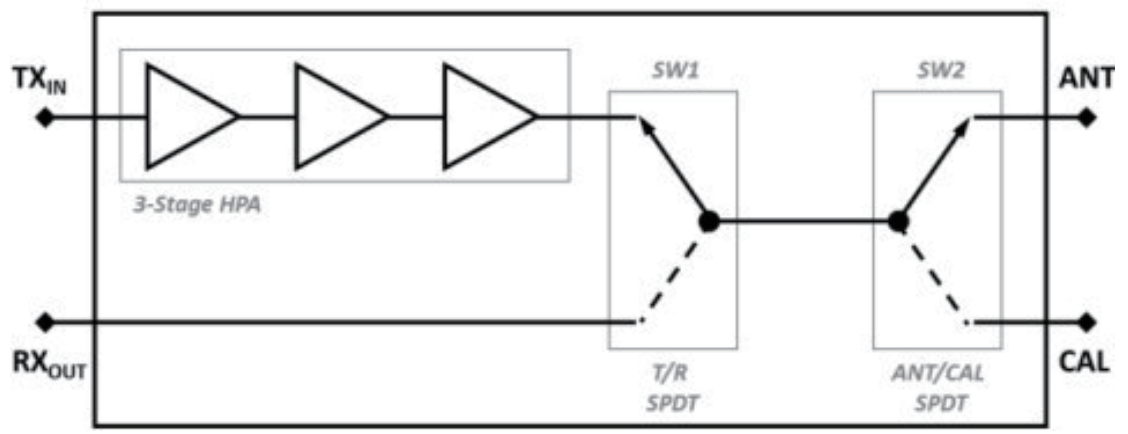

Fig. 1. Block diagram of conventional RF front-end system.

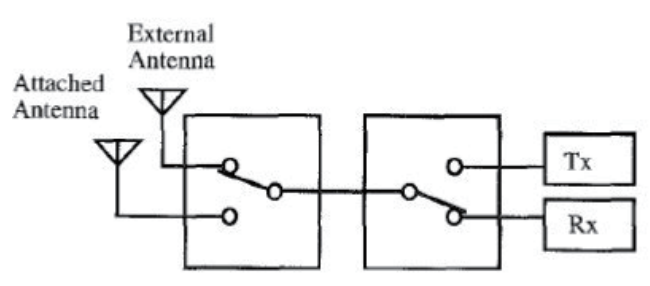

(a)

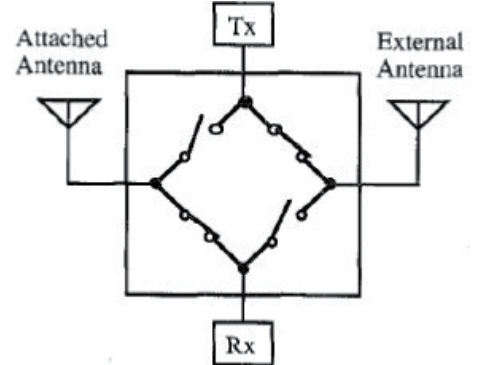

(b)

Fig. 2. (a) Conventional DPDT switch. (b) Ring-type DPDT switch.

series microstrip lines to form a transmission line with a characteristic impedance of $50 \Omega$. The isolation was better than $25 \mathrm{~dB}$ from dc to $60 \mathrm{GHz}$. Using the transmission-line integrated approach and body-bias technique, ${ }^{(4)}$ the switch achieved high isolation even beyond $100 \mathrm{GHz}$. Based on double-shunt transistors with quarter-wavelength transmission lines, ${ }^{(5)}$ the switch had a measured isolation of $39 \mathrm{~dB}$ at $60 \mathrm{GHz}$. Recently, eighth-wavelength transmission lines have been proposed to replace quarter-wavelength lines in order to improve the performance of circuits. ${ }^{(6)}$ A wide-bandwidth high-frequency switch with a compact size has demonstrated stable isolation. ${ }^{(7)}$ Recently, research on RF switches for wireless sensors has proved their feasibility.

In this paper, we propose a new structure for a DPDT switch using the traveling-wave concept with eighth-wavelength transmission lines. Compared with the performance of conventional quarter-wavelength SPDT and DPDT switches, the isolation reaches a value as high as $29 \mathrm{~dB}$. This new structure can help to improve the isolation performance in sensor applications.

\section{Materials and Methods}

The proposed novel DPDT circuit is designed using a ring-type structure. As shown in Fig. 3(c), the switch has a three-stage structure with eighth-wavelength transmission lines. The 


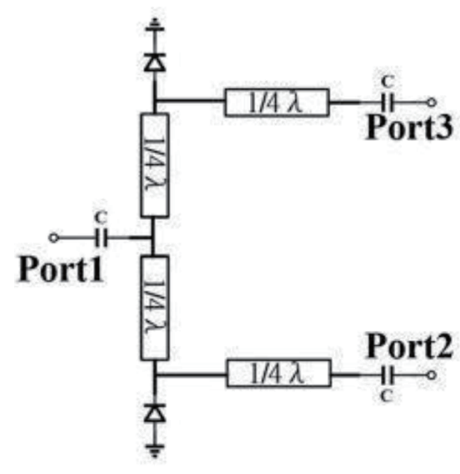

(a)

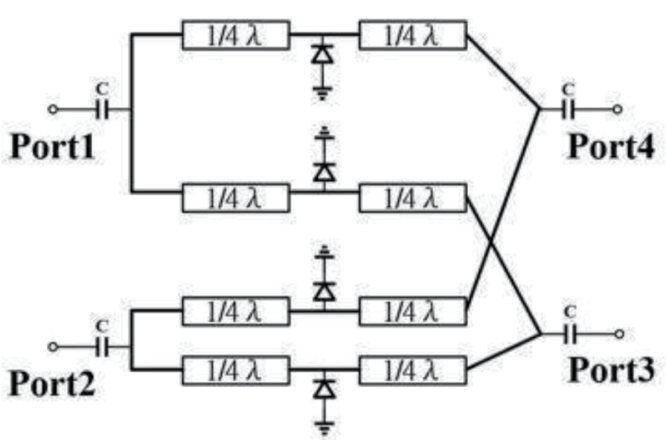

(b)

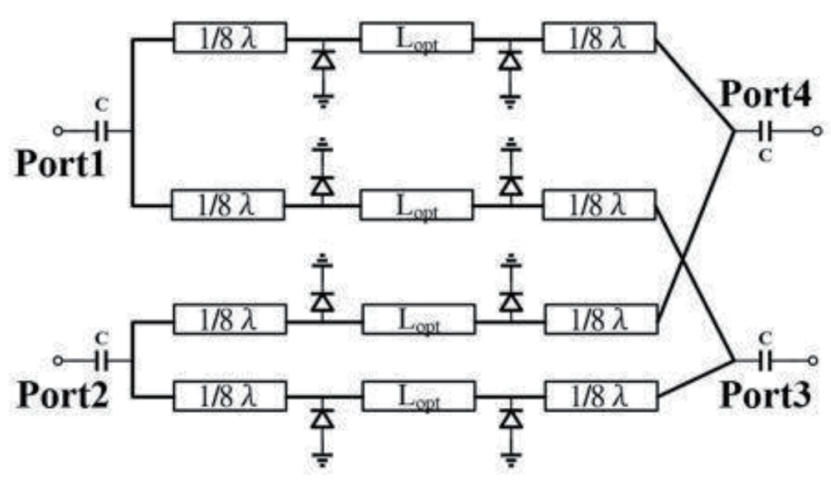

(c)

Fig. 3. Conventional quarter-wavelength (a) SPDT and (b) DPDT switches, and (c) eighth-wavelength DPDT switch.

diodes between stages are used for switching on and off. The eighth-wavelength transmission lines are used as inductors, and the transmission lines Lopt are optimized at the working frequency of $2.4 \mathrm{GHz}$. If one of the four ports is connected to a $50 \Omega$ impedance to the ground, the switch can be used as an SPDT switch. Also, the schematics of the conventional quarterwavelength SPDT and DPDT switches are respectively shown in Figs. 3(a) and 3(b).

When given a bias voltage of $7.5 \mathrm{~V}$, half of the diodes are equivalent to the capacitors and the other half are equivalent to the resistors. The equivalent circuit is shown in Fig. 2. For example, if the path from portl to port 4 is in the ON state, the path from portl to port3 is an open circuit and works as a low-pass filter, which absorbs the signals from the path. Figure 4 shows the equivalent $\mathrm{ON}$ and $\mathrm{OFF}$ state circuits of the switch at the working frequency of $2.4 \mathrm{GHz} . \mathrm{C}_{1}$ and $\mathrm{R}_{1}$ are used in the analysis for simplicity. Also, the inductors are in the approximate state of transmission lines at the specific frequency. Figure 5 shows the equivalent circuit of the proposed switch when the circuit is switching on and off.

In contrast to the conventional single-stage quarter-wavelength SPDT and DPDT switches, this eighth-wavelength DPDT switch uses a multistage structure to enhance the isolation performance, which has been demonstrated elsewhere. ${ }^{(6)}$ 


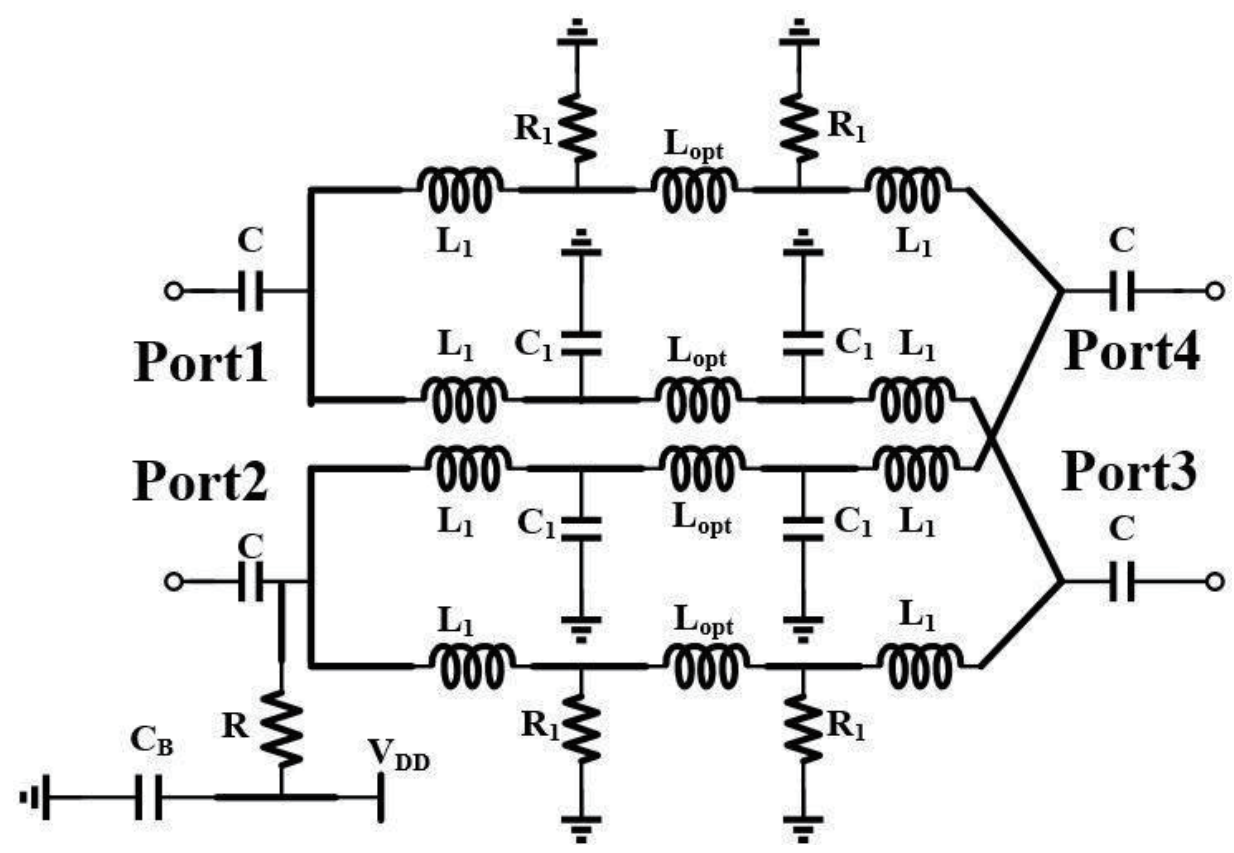

Fig. 4. Equivalent circuit of switch in $\mathrm{ON}$ and OFF states.

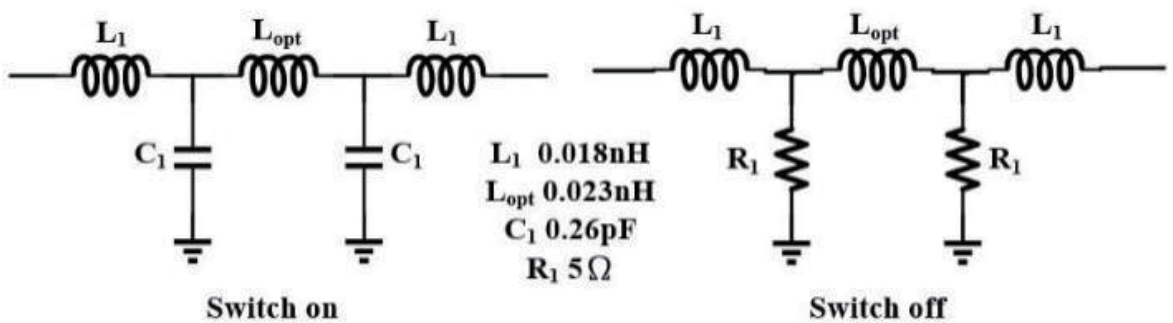

Fig. 5. Equivalent circuit of proposed switch.

To improve the performance and enable printed circuit board (PCB) etching, the transmission line width needs to be optimized. We simulated the proposed switch with line widths of 0.25 , 0.5 , and $1 \mathrm{~mm}$ with the bias voltage set at $7.5 \mathrm{~V}$. Figure 6 shows the simulated insertion loss and isolation for the different line widths. The simulation results show that the insertion loss is nearly the same for the different line widths. However, a smaller line width results in a higher isolation. We choose $0.5 \mathrm{~mm}$ as the line width in consideration of the etching process. The width and length of a microstrip line are calculated as follows:

$$
\frac{W}{d}=\left\{\begin{array}{cc}
\frac{8 e^{A}}{e^{2 A}-2} & \text { for } \frac{W}{d}<2, \\
\left.\frac{2}{\pi}\left[B-1-\ln (2 B-1)+\frac{\varepsilon_{r}-1}{2 \varepsilon_{r}}\left\{\ln (B-1)+0.39-\frac{0.61}{\varepsilon_{r}}\right\}\right]\right] & \text { for } \frac{W}{d}>2,
\end{array}\right.
$$




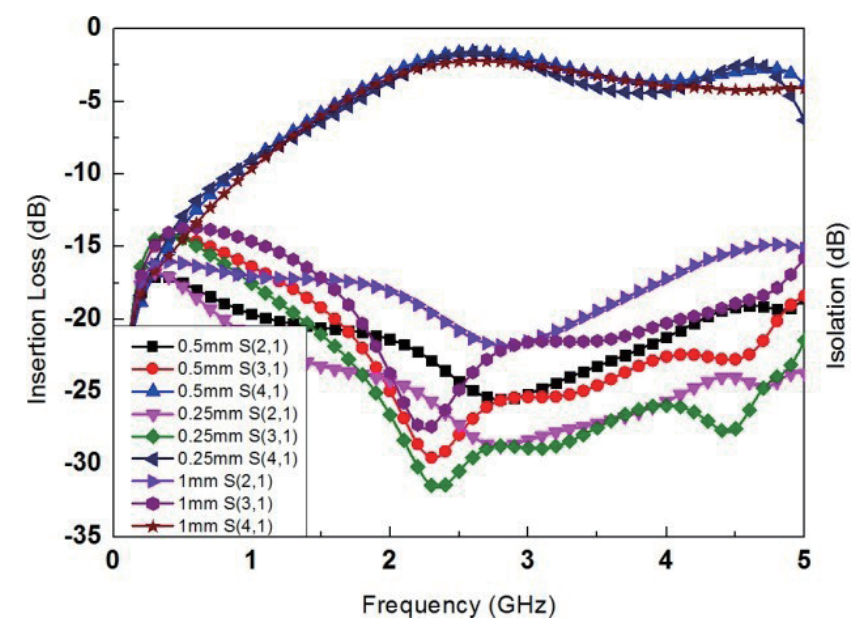

Fig. 6. (Color online) Insertion loss and isolation for different line widths.

Table 1

Circuit parameters of DPDT switch.

\begin{tabular}{lc}
\hline Components & Parameters \\
\hline TL $1 / 8 \lambda$ & Length $=7 \mathrm{~mm}$, Width $=0.5 \mathrm{~mm}$ \\
TL L opt $_{C}$ & Length $=9 \mathrm{~mm}$, Width $=0.5 \mathrm{~mm}$ \\
$C / C_{B}$ & $20 \mathrm{pF} / 3 \mathrm{pF}$ \\
$R$ & $1000 \Omega$ \\
\hline
\end{tabular}

$$
\begin{gathered}
A=\frac{Z_{0}}{60} \sqrt{\frac{\varepsilon_{r}+1}{2}}+\frac{\varepsilon_{r}-1}{\varepsilon_{r}+1}\left(0.23+\frac{0.11}{\varepsilon_{r}}\right), \\
B=\frac{377 \pi}{2 Z_{0} \sqrt{\varepsilon_{r}}}, \\
l=\frac{90^{\circ}\left(\pi / 180^{\circ}\right)}{\sqrt{\varepsilon_{e}} K_{0}},
\end{gathered}
$$

where $W$ is the width of the microstrip line in $\mathrm{mm}, l$ is the length of the microstrip line in $\mathrm{mm}$, and $d$ is the thickness of the FR4 substrate in $\mathrm{mm} ; K_{0}=(2 \pi f) / c$. The parameters of the novel DPDT switch are shown in Table 1.

\section{Measured Results}

The proposed DPDT switch was fabricated using an FR4 PCB, and the Schottky diode HSMS-2860 was fabricated by the CMOS process. Figure 7 shows images of the fabricated switches. The chip size of the novel eighth-wavelength switch is $4.5 \times 5 \mathrm{~cm}^{2}$. Also, for 


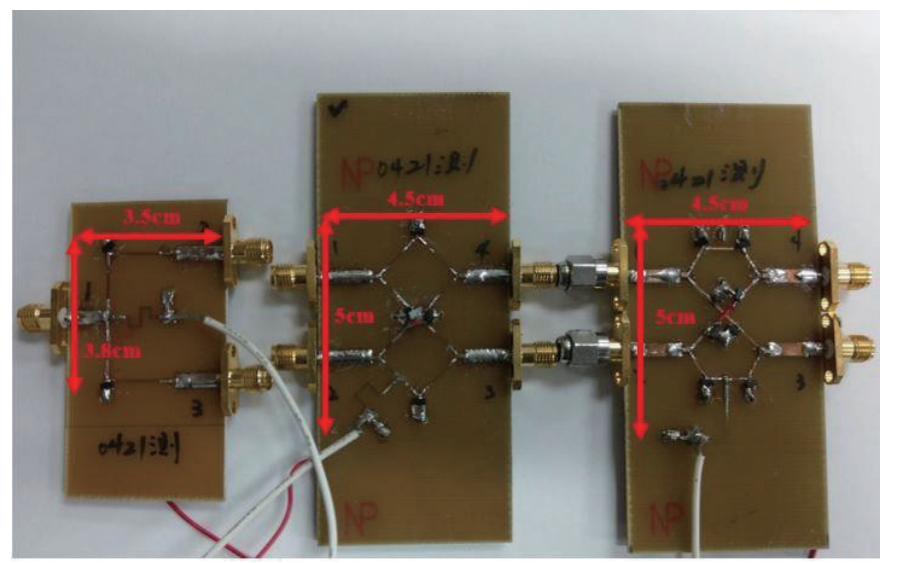

(a)

(b)

(c)

Fig. 7. (Color online) Circuit photo of (a) conventional SPDT and (b) DPDT switches, and (c) novel DPDT switch.

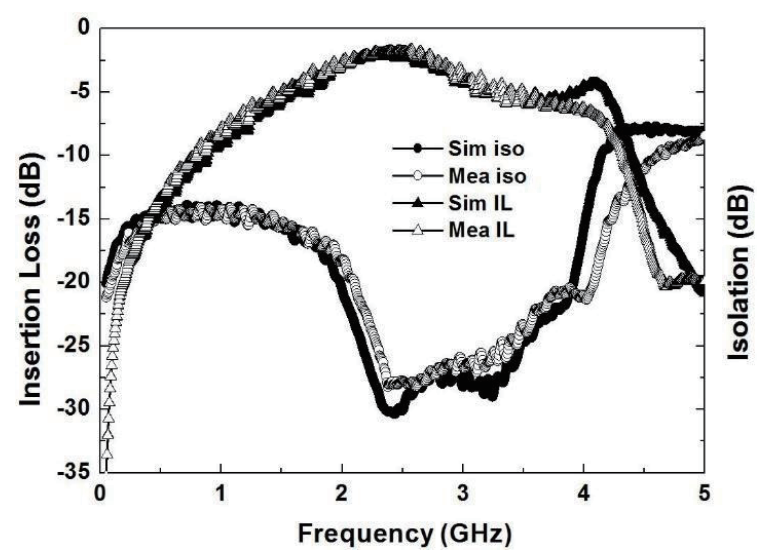

Fig. 8. Insertion loss and isolation of proposed switch.

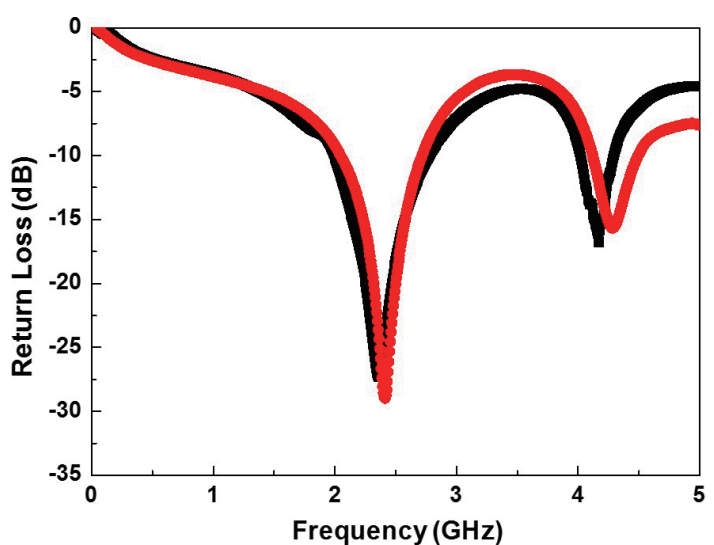

Fig. 9. (Color online) Return loss of proposed switch.

comparison, the conventional quarter-wavelength SPDT and DPDT switches are shown in Figs. 7(a) and 7(b), respectively.

An Agilent E8364A parameter network analyzer (PNA) was used to measure the S-parameter of the three switches. The IF bandwidth was $200 \mathrm{~Hz}$ and the S-parameter was measured from $50 \mathrm{MHz}$ to $5 \mathrm{GHz}$ at 401 points. Because the PNA can only measure two ports, we installed two $50 \Omega$ dummy resistors on the two other ports. An Agilent E3648A power supply was used to provide a bias voltage of $7.5 \mathrm{~V}$ and the current was limited to $9 \mathrm{~mA}$.

The measured insertion loss of the novel DPDT switch was $1.65 \mathrm{~dB}$ and the isolation was $29 \mathrm{~dB}$ at the working frequency of $2.4 \mathrm{GHz}$ as shown in Fig. 8. The input and output return losses were larger than $25 \mathrm{~dB}$ at the working frequency as shown in Fig. 9.

For comparison, the conventional quarter-wavelength SPDT and DPDT switches were also measured. The insertional losses of the quarter-wavelength SPDT and DPDT switches and the eighth-wavelength DPDT switch were 1.07, 1.6, and $1.65 \mathrm{~dB}$ at the frequency of $2.4 \mathrm{GHz}$, 


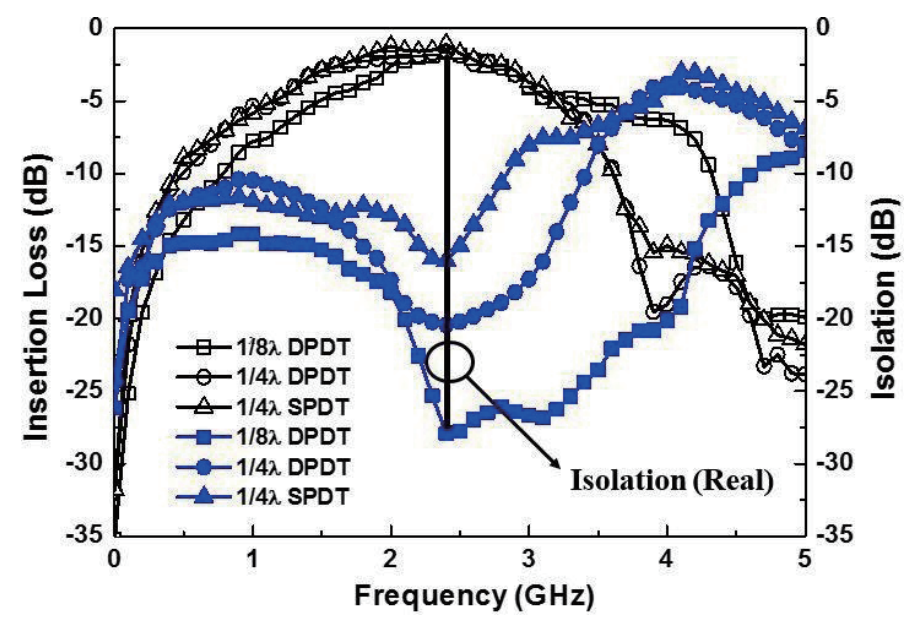

Fig. 10. (Color online) Insertion loss and isolation of three switches.

Table 2

Comparison of three switches.

\begin{tabular}{lccc}
\hline Spec. & Novel DPDT switch & $\begin{array}{c}\text { Quarter-wavelength } \\
\text { DPDT switch }\end{array}$ & $\begin{array}{c}\text { Quarter-wavelength } \\
\text { SPDT switch }\end{array}$ \\
\hline Insertion loss $(\mathrm{dB})$ & 1.65 & 1.6 & 1.07 \\
Isolation $(\mathrm{dB})$ & 29 & 21 & 16 \\
Area $\left(\mathrm{cm}^{2}\right)$ & $4.5 \times 5$ & $4.5 \times 5$ & $3.5 \times 3.8$ \\
\hline
\end{tabular}

respectively. The measured isolations for the three switches were 16,21 , and $29 \mathrm{~dB}$, respectively, as presented in Fig. 10. For the DPDT switch with the ring-type structure, the real isolation was defined as the measured isolation minus the insertion loss. Table 2 provides a comparison of the three switches. This novel eighth-wavelength DPDT switch has the best isolation among the switches, while the insertion loss and area are nearly the same as those of the quarterwavelength DPDT switch.

\section{Conclusion}

In this paper, a novel DPDT switch using eighth-wavelength transmission lines was presented. The positive and negative bias voltages of $7.5 \mathrm{~V}$ are used for switching the switch on and off. There was some variation in the measurement due to the jump wire in the middle of the PCB. The measured result shows that the switch has the best isolation of $29 \mathrm{~dB}$ among the three switches while the insertion loss was $1.65 \mathrm{~dB}$. The results for this switch have demonstrated its effectiveness and potential in wireless sensor applications.

\section{Acknowledgments}

This work was supported by Fundamental Research Funds for the Central Universities, JUSRP12023. 


\section{References}

1 W. Xia, Y. Wen, C. Foh, D. Niyato, and H. Xie: IEEE Commun. Surv. Tutorials 17 (2015) 27.

2 M. Donelli and J. Iannacci: IEEE Antennas Wirel. Propag. Lett. 18 (2019) 44.

3 K. Y. Lin, W. H. Tu, P. Y. Chen, H. Chen, H. Wang, and R. B. Wu: IEEE Trans. Microw. Theory Tech. 52 (2004) 1798.

4 R. B. Lai, J. J. Kuo, and H. Wang: IEEE Microw. Wirel. Compon. Lett. 20 (2010) 85.

5 C. Byeon and C. S. Park: IEEE Trans Microw. Theory Tech. 61 (2013) 2858.

6 R. Zhang, S. Luo, and L. Zhu: IEEE Microw. Wirel. Compon. Lett. 24 (2014) 680.

7 L. Zhao, W. F. Liang, J. Y. Zhou, and X. Jiang: IEEE Microw. Wirel. Compon. Lett. 27 (2017) 485. 